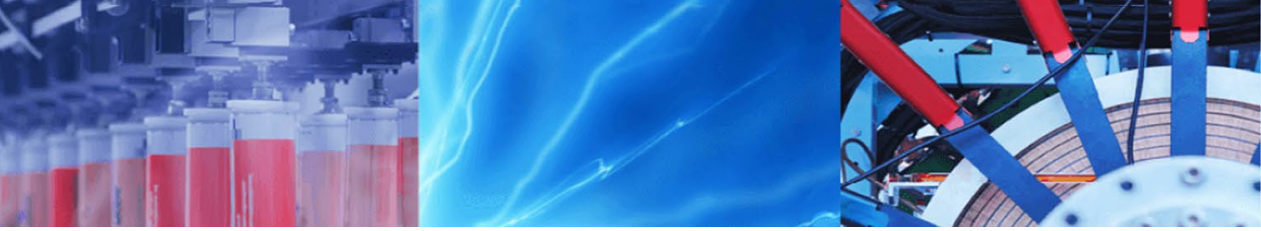

Short Communication

\title{
Design aspects of (super)hydrophobic mesh based oil-collecting device with improved efficiency
}

\author{
Weikang Xu' ${ }^{1}$ Zhentao Zhang ${ }^{1} \cdot$ Xiaomei Cai ${ }^{2} \cdot$ Yazhen $\mathrm{Hong}^{2} \cdot$ Tianliang Lin $^{1} \cdot$ Tingping Lei $^{1}$
}

Received: 3 September 2020 / Accepted: 7 January 2021 / Published online: 13 January 2021

(C) The Author(s) 2021 OPEN

\begin{abstract}
Effective treatment of frequent oil spills and endless discharged oily wastewater is crucial for the ecosystem and human health. In the past two decades, the collection of oil from water surface has been widely studied through the simple fabrication of superhydrophobic meshes with various coating materials, but little attention is paid to the design aspects of the meshes based oil-collecting device and practical oil collection. Here, 3D-printing devices with different configurations of (super)hydrophobic meshes, circular truncated cone (CTC), cylinder and inverted CTC, and the same inverted cone-shaped structure (below the meshes for temporary oil storage) are investigated. Results demonstrate that the CTC meshes based device especially for an oblate one not only shows higher stability and discharge of the collected oils than previous reports, but also allows floating oils to enter the (super)hydrophobic mesh faster. We anticipate that future success in developing high-performance (super)hydrophobic meshes and the further optimization of the CTC mesh-based device parameters will make our proposed device more practical for the treatment of real-life oil spills.
\end{abstract}

Keywords Oil/water separation · Floating oil collection · Superhydrophobic mesh · Circular truncated cone (CTC) structure $\cdot 3 \mathrm{D}$-printing

\section{Introduction}

The frequent oil spill accidents and discharged oily wastewater not only cause serious environmental pollution, but also impose severe threats to human health and the ecosystem [1, 2]. Traditional techniques, such as in situ burning [3], skimmers [4], centrifugation [5], air flotation [6], and biological/chemical methods [7], have been utilized to treat oily wastewater. However, these countermeasures normally suffer from low separation efficiency, high cost, and some even bring secondary pollution, urging researchers to develop more effective strategies [8-10].

Since oil/water separation is essentially an interfacial issue, researchers have turned to interfacial superwetting porous materials to treat oily water. In 2004 , the superiority of a superhydrophobic and superoleophilic coating mesh to separate oil/water mixtures was first demonstrated [11]. Since then, oil/water separation based on materials with special wettability, through either filtration or absorption, has gained great interest. In terms of the absorption, naturally amphiphilic (oil-wet and water-wet) sponges [12, 13] or foams [14, 15] are made hydrophobic to absorb oils from oil/water mixtures. However, repeated squeezing or simultaneous external pumping inevitably causes water mixed in the absorbed oils [16-19]. As for the filtration, metal mesh is made superhydrophobic or underwater superoleophobic and integrated into a filtration device for oil/water separation [20-22], where oil/water mixtures are necessarily transferred into the filtration device, such that more time and labor are consumed. Interestingly, superhydrophobic filtration device can be also directly used to collect oils from oil/water mixture when

$\triangle$ Xiaomei Cai, cxplum@163.com; $\bowtie$ Tingping Lei, tplei@hqu.edu.cn| ${ }^{1}$ College of Mechanical Engineering and Automation, Huaqiao University, Xiamen 361021 , China. ${ }^{2}$ School of Science, Jimei University, Xiamen 361021, China. 
a superhydrophobic two-dimensional (2D) mesh or foam is folded into a three-dimensional (3D) box-like structure (also called "mini-boat") [23, 24]. Through further external pumping, continuous oil collection can be realized [22, 25-28]. However, such a "mini-boat" is vulnerable in collection of spilled oils in real-life marine environment. To the best of our knowledge, currently the development of various superhydrophobic materials [29-31] still receive great interest, and little or no attention is paid to the structure/ shape of the superhydrophobic materials coated meshes/ membranes and their related device configurations, which is significant for improving the collection efficiency as well as for the practical use.

In this work, design aspects of an efficient oil-collecting device based on (super)hydrophobic meshes are discussed. Experimental results demonstrate that a proper configuration/structure of the device can much improve the oil collection, allowing the floating oils to enter the (super)hydrophobic mesh faster as well as showing higher stability and discharge of the collected oils.

\section{Experimental}

Polyvinylidene fluoride (PVDF, D692) powder was purchased from Shanghai Sensure Chemical Co. Ltd. (China). Hydrophobic silica nanoparticles $\left(\mathrm{SiO}_{2}, \mathrm{XZ}-\mathrm{G} 02\right)$ were provided by Hefei Xiang Zheng Chemical Technology Co. Ltd. (China). Acetone (analytical reagent) and N, N-dimethylformamide (DMF, analytical reagent) were supplied by Sinopharm Chemical Reagent Co., Ltd. (China). Kerosene, $n$-hexadecane and petroleum ether were provided by Shanghai Aladdin Bio-Chem Technology Co., Ltd. (China). These solvents were chemical grade and were used without further purification.

The coating material was prepared by adding $3.0 \mathrm{~g}$ $\mathrm{SiO}_{2}$ nanopowder and $2.0 \mathrm{~g}$ PVDF powder (a raw material that we have intensively studied previously $[32,33]$ ) to the mixed solvents of $60 \mathrm{ml} \mathrm{DMF}$ and $40 \mathrm{ml}$ acetone with continuous magnetic stirring at room temperature for $8 \mathrm{~h}$ to form $36.2 \%$ cocktail of PVDF-SiO ${ }_{2}$. The dip coating method was employed to fabricate superwetting PVDF-SiO ${ }_{2}$ meshes. Specifically, the acetone- and ethanoltreated Cu meshes were dipped into the as-prepared cocktail for $5 \mathrm{~min}$ and air-dried under ambient atmosphere. If not stated otherwise, the mesh number for Cu mesh is 120 .

The surface morphology of the as-prepared meshes was observed using field-emission scanning electron microscopy (FE-SEM, Zeiss, Sigma-HD-01-36, Germany). The wettability was characterized by a contact angle analyzer (JC2000D3, Shanghai, China) using a droplet $(9 \mu \mathrm{l})$ of water or hexane as an indicator at room temperature. The contact angle data were figured out based on ellipse fitting method and the final result was averaged from five measurements for each sample.

The oil collection experiment was conducted using our designed collecting devices, which are detailed in the following section. Unless expressly stated otherwise, $n$-hexadecane $\left(0.77 \mathrm{~g} / \mathrm{cm}^{3}\right)$ was used as the light oil to form an oil slick, and the amount was $150 \mathrm{~g}$ that approximately equals $5 \mathrm{~mm}$ oil slick on the water surface as determined by the tank. During the collecting process, a pumping rate of $200 \mathrm{~mL} / \mathrm{min}$ was selected to guarantee the collected oils to be pumped away immediately. A digital electronic scale was used to record the real-time amount of the collected oils in the container.

\section{Results and discussion}

Figure 1 shows the schematic (A) and picture (B) of (super) hydrophobic mesh-based oil-collecting device that can be used for continuous collection of the floating oils with several advantages. Unlike previous reports where (super) hydrophobic meshes are folded into tube-shape structures or various mini-boats [23-25], the mesh here is tailored
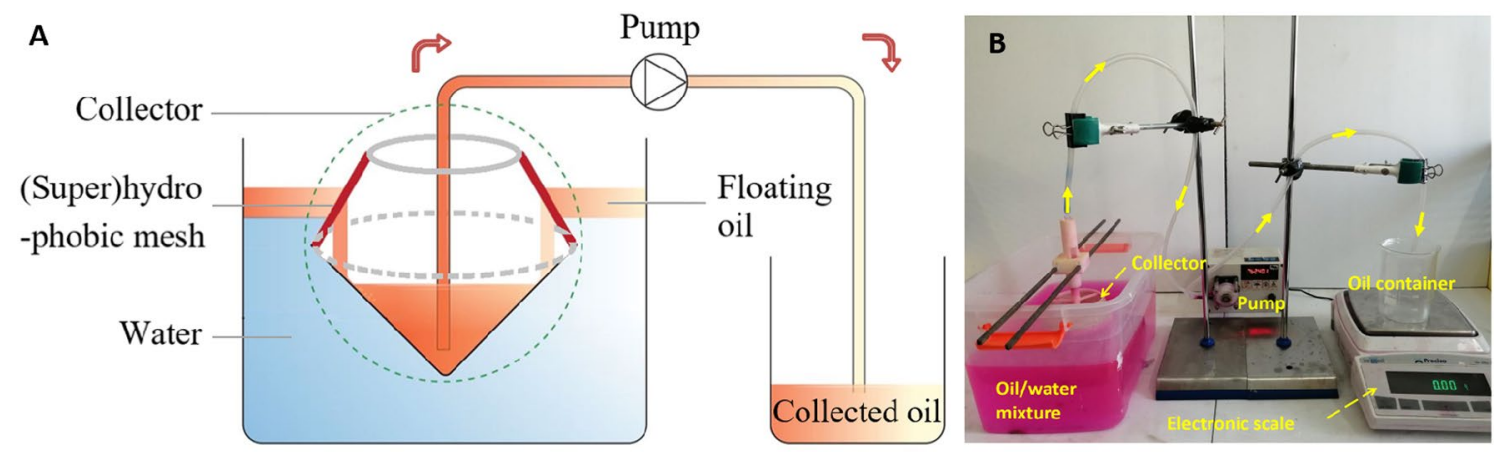

Fig. 1 a Schematic and (b) photo of proposed oil-collecting device for continuous collection of the floating oils. Note (super)hydrophobic mesh here is tailed into a circular truncated cone (CTC) structure instead 
into a circular truncated cone (CTC) structure. Such a structure allows the easier penetration of the floating oils into the mesh and thus accelerates the oil collection process, which is detailed in the next paragraph. As shown in Fig. 1a, the lower part of the collector (below the CTC mesh) is an inverted cone-shaped structure used for temporary oil storage. This inverted cone structure not only enables the collector to keep stable in practical oil collection [34], but also facilitates the evacuation of temporarily collected oils in spite of little amount of the oils [22]. By employing a pump that is connected with some pipes, consecutive transportation of the oils to the container can be easily realized.

As mentioned in the introduction, the (super)hydrophobic-mesh-based oil-collecting device configuration/ structure especially the shape of the meshes should more or less affect the oil collection efficiency. Here, the meshes in three typical configurations, a CTC, a cylinder and an inverted CTC have been designed and compared.
For the case of light oils permeating through the superhydrophobic mesh, the oils on the sidewall will have different force conditions. As shown in Fig. 2a, the gravity force $\left(F_{\mathrm{g}}\right)$ in the CTC (left) has a $y$-directional component $\left(F_{g, y}^{\prime}\right)$, such that the permeation of the oils to the sidewall is strengthened to assist the passage of the floating (light) oils. In contrast, $F_{\mathrm{g}}$ in the inverted CTC (right), has an opposite $y$-directional component $\left(-F_{g, y}^{\prime}\right)$ to slow down the passage of the floating oils, whereas $F_{\mathrm{g}}$ in the cylinder (middle) remains "neutral" to the passage of the floating oils. The above theoretical analysis is also supported by the experiment of light oil collection from the same oil/water mixture (150 $\mathrm{g}$ n-hexadecane equaling to the oil slick thickness of $5 \mathrm{~mm}$ ) and a constant pumping rate of $200 \mathrm{~mL} / \mathrm{min}$ (enough to pump away the collected oils immediately) (Fig. 2b). It should be noted that the initial contact area of mesh and oil slicks is the same for the three configurations, as illustrated in Fig. 2c.
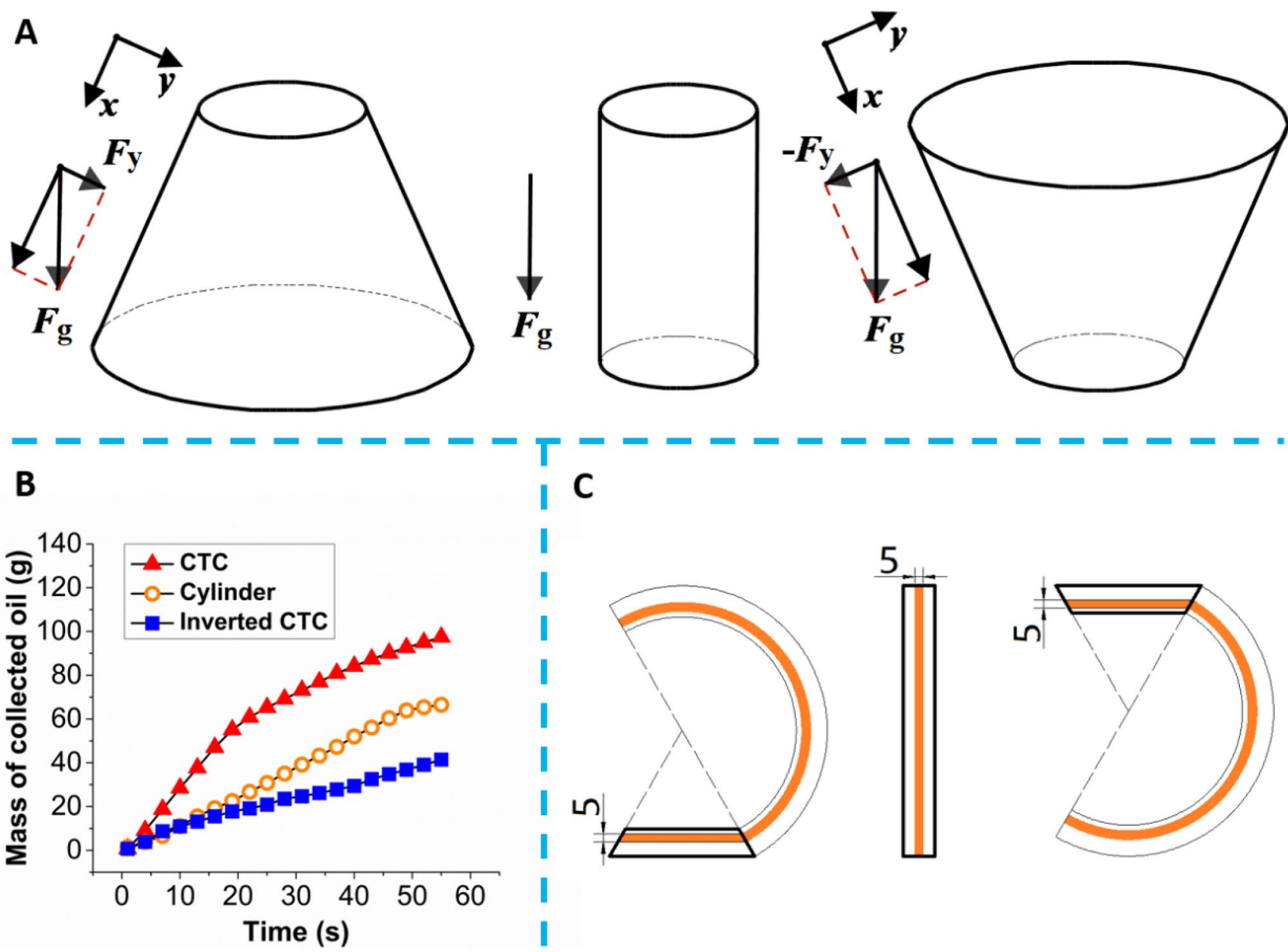

Fig. 2 a Effect of oil gravity on its passage through the sidewall of circular truncated cone (CTC), cylinder and inverted CTC (super) hydrophobic meshes. b Collected $n$-hexadecane $(150 \mathrm{~g}$, oil slick thickness: $5 \mathrm{~mm}$ ) from oil/water mixture (pumping rate: $200 \mathrm{~mL}$ / min) by the three typical structures. c Side and unfolded view of meshes integrated on the three structures and actual contact areas, numerically equal ( $1256.64 \mathrm{~mm}^{2}$, highlighted by orange color), between oil and meshes 
From the above theoretical analysis and experimental result, it is clear that by tailoring (super)hydrophobic mesh into a CTC-shaped structure the oil collection is much improved. Also from Fig. 2a (left), the $y$-directional component $\left(F_{g, y}^{\prime}\right)$ of gravity force is closely related to the inclination angle of the CTC, such that the collection rate of different angles should be different. Here, CTC meshes with the typical inclination angles of $30^{\circ}, 60^{\circ}$ and $75^{\circ}$ are compared. For this purpose, frameworks of them were precisely machined via 3D printing, as shown in Fig. 3a-c. Figure $3 \mathrm{~d}-\mathrm{f}$ shows the CTC frameworks with (super)hydrophobic meshes of the same coating materials partially immersing in the water, which reveals a sharper concave meniscus for the one with smaller inclination angle. To observe this phenomenon enough clearly especially for a big inclination angle (e.g. $75^{\circ}$ ), the water was dyed (Fig. $3 f$ ). Under the same thickness of oil slick, the same actual contact area of oil/mesh and a pumping rate of $200 \mathrm{~mL} /$ $\mathrm{min}$, the collector (oblate) with an inclination angle of $30^{\circ}$ shows the best collecting rate (which may be due to the sharpest concave meniscus in Fig. 3d) although in the first $15 \mathrm{~s}$ both " $30^{\circ}$ " and " $60^{\circ}$ " show the similar result due to experimental uncertainty (Fig. 4a). The above experimental result demonstrates that with the inclination angle decreased the collecting rate normally increases, which is agreeable with the force analysis in Fig. 2a (left).

Figure $4 \mathrm{~b}-\mathrm{d}$ shows the result of using the above-mentioned three typical CTC-shaped collectors to collect the floating oils from water surface under "dynamic" (waving) and "static" (non-waving) conditions. Here "dynamic" and "static" correspond to the as-prepared light oil/water mixture with and without continuous stirring, respectively. It is clear that all the collectors exhibit higher oil collecting rate under dynamic condition except for the first few seconds. The reason is that the waving water surface normally accelerates the floating oils to approach to the mesh and therefore more oils are collected during the same period. However, it is noted that in the initial stage of collection, the wetting process of the mesh, the temporary storage in the collector and transportation through the pipe (Fig. 1) should take some time, such that there is tiny difference in the first few seconds (Fig. 4b-d). Interestingly, as the inclination angle increases the difference of the oil collection under dynamic and static conditions becomes increasingly distinct, as indicated in Fig. 4b-d.

Figure 5 demonstrates the process of floating oil collection via a CTC-shaped collector with an inclination assngle of $30^{\circ}$. A little amount of $n$-hexadecane (dyed in blue) was gradually poured into the water, and it was found the oil drops quickly swam towards the (super)hydrophobic mesh and permeated through the mesh successively. Hence, floating oil collection can be realized by simply placing the collector on water surface. However, it should be noted that if there was no collector in the water, the oil drops were nearly static and kept still for a long time (Figure not shown here).Apart from the consideration of collector
Fig. 3 a-c Frameworks of 3D-pringting CTC-shaped collectors with different inclination angles $\left(30^{\circ}, 60^{\circ}\right.$ and $\left.75^{\circ}\right)$ as illustrated. $\mathbf{d}-\mathbf{f}$ Phenomena of hydrophobicity on mesh surfaces with different inclination angles $\left(30^{\circ}, 60^{\circ}\right.$ and $75^{\circ}$, respectively)
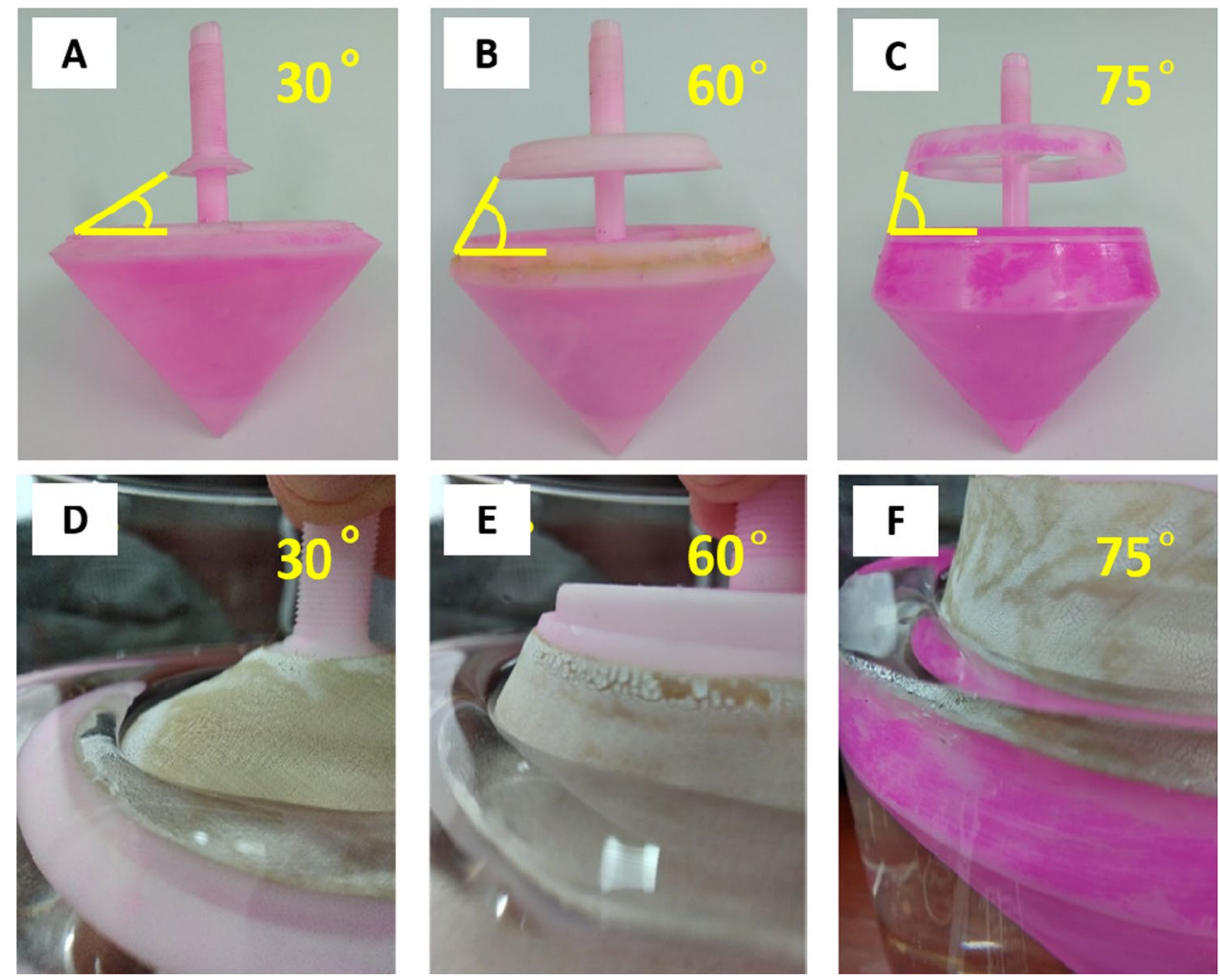

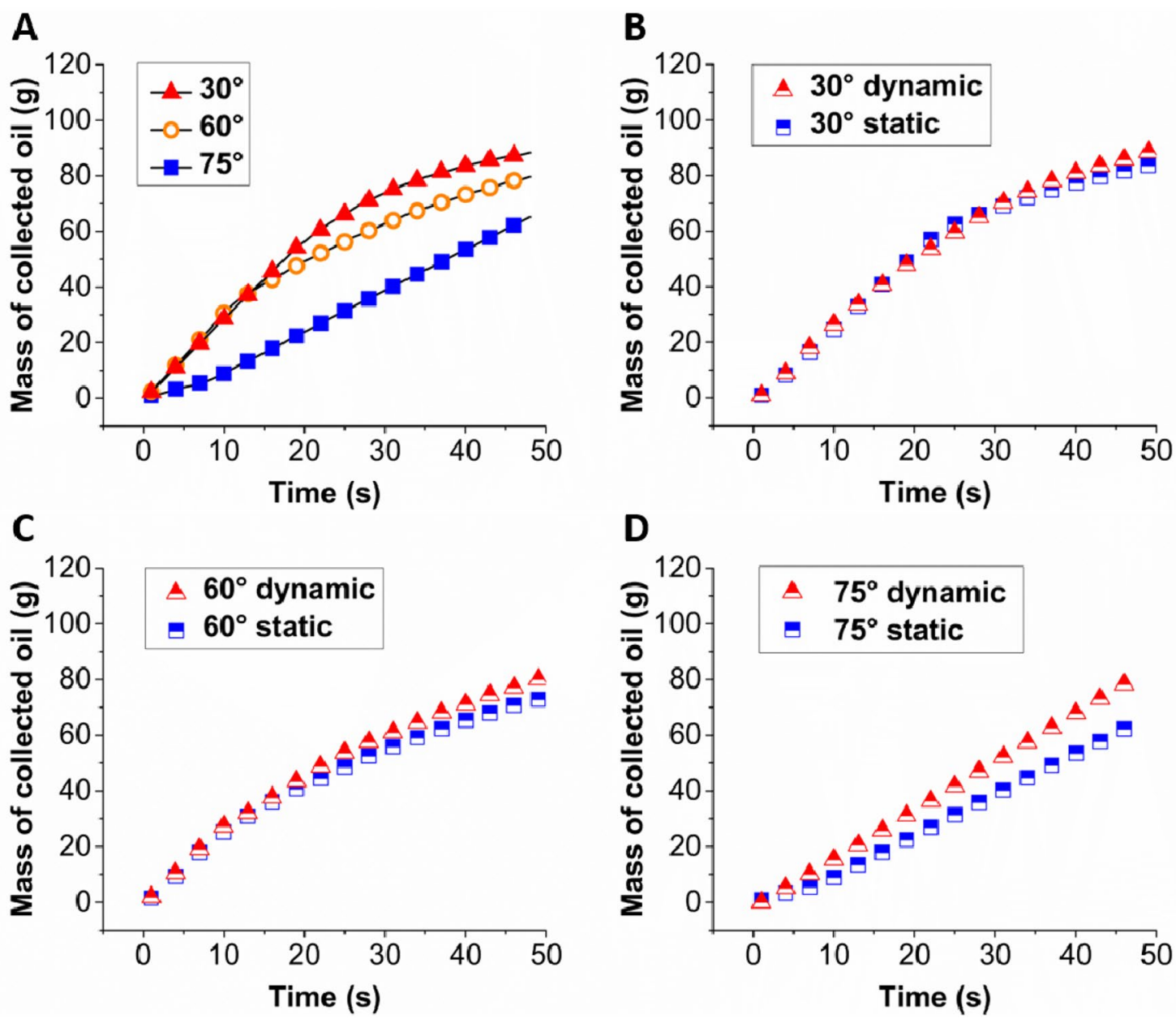

D

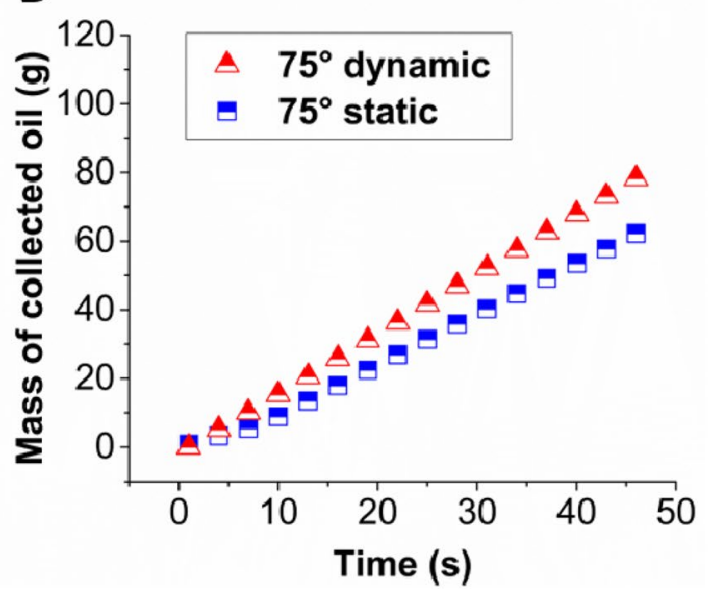

Fig. 4 a Mass of the collected $n$-hexadecane versus time using the above-mentioned CTC-shaped collectors. b-d Comparison of the collected $n$-hexadecane under the conditions of "dynamic" and

"static" water surface using the above-mentioned CTC-shaped collectors. The "dynamic" water surface is simulated by a magnetic stirrer
Fig. 5 a Front view and (b) top view of floating oil (dyed blue) collection via the $30^{\circ}$ CTC-shaped collector without pumping
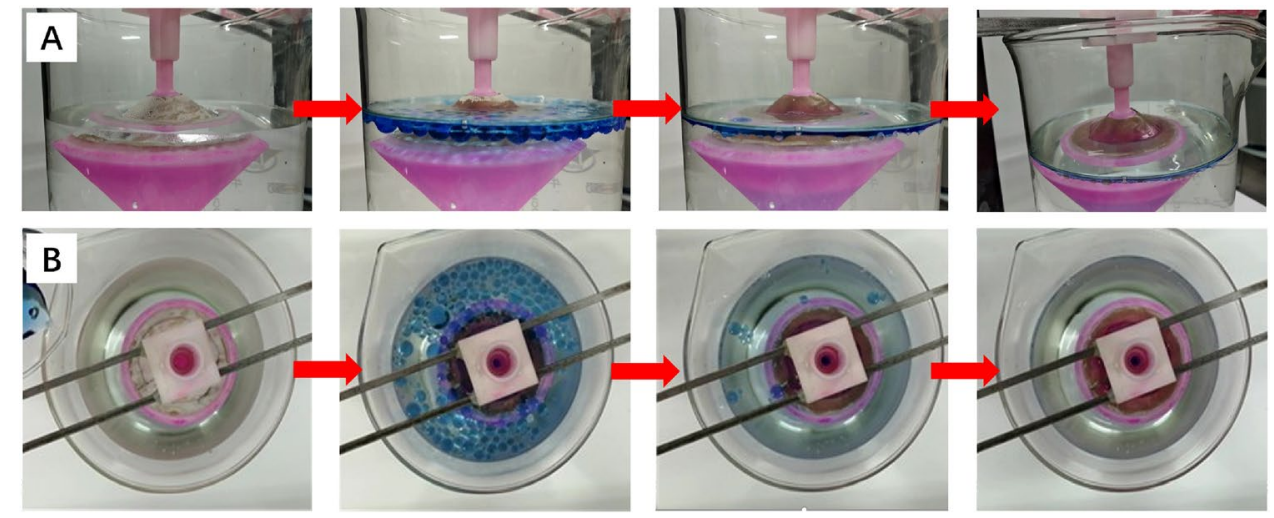

configuration, the mesh number, mesh substrate material, the amount of floating oil (thickness of oil slick), and the type of light oil were also investigated for the CTC-shaped collector with inclination angle of $60^{\circ}$. Figure 6a shows that as the mesh number decreases from 200 to 120 , the collecting rate increases, which can be attributed to the difference of pore size [35]. For the same mesh number (200) of the different substrates, $\mathrm{PVDF} / \mathrm{SiO}_{2}$-coated stainless steel mesh shows a higher collecting rate than copper mesh of the same coating especially after $20 \mathrm{~s}$ (Fig. 6b). 
A

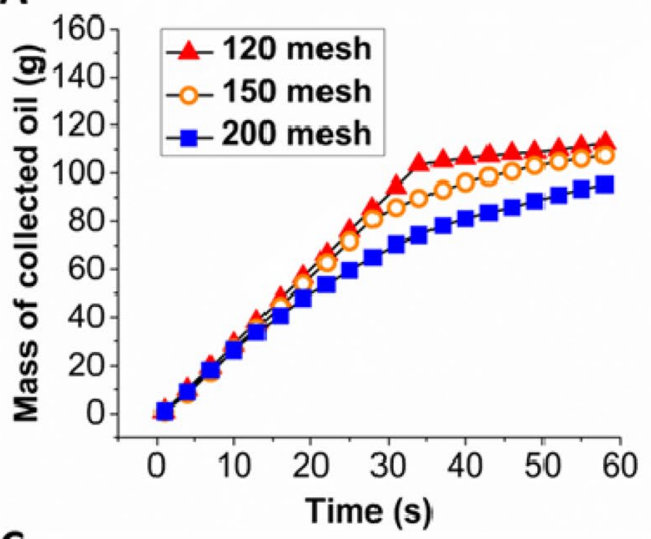

C

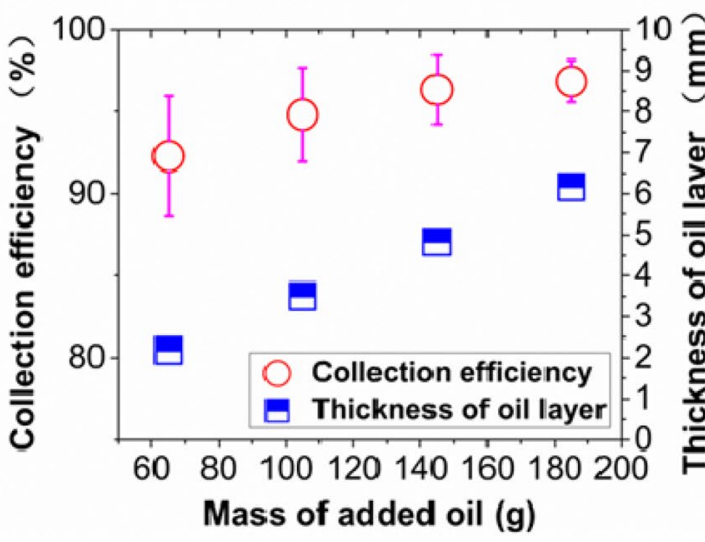

E

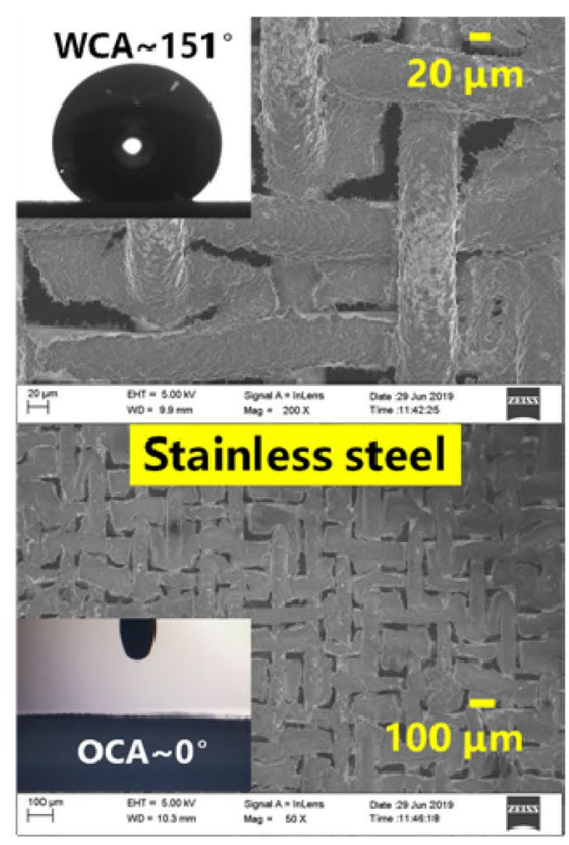

The reason is that the micro structure and roughness of the two coated meshes are different as revealed from the SEM micrographs in Fig. 6e. The WCA of modified stainless steel mesh reached $151^{\circ}$ while the OCA was $0^{\circ}$. Similarly, the water droplet on the modified copper mesh reached
B

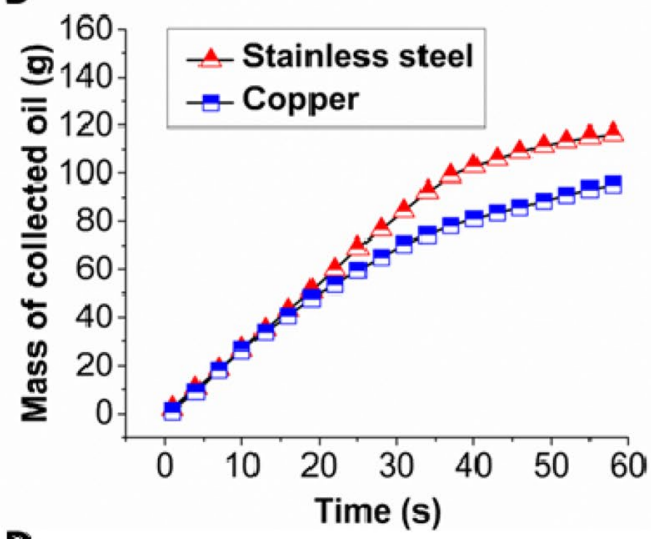

D
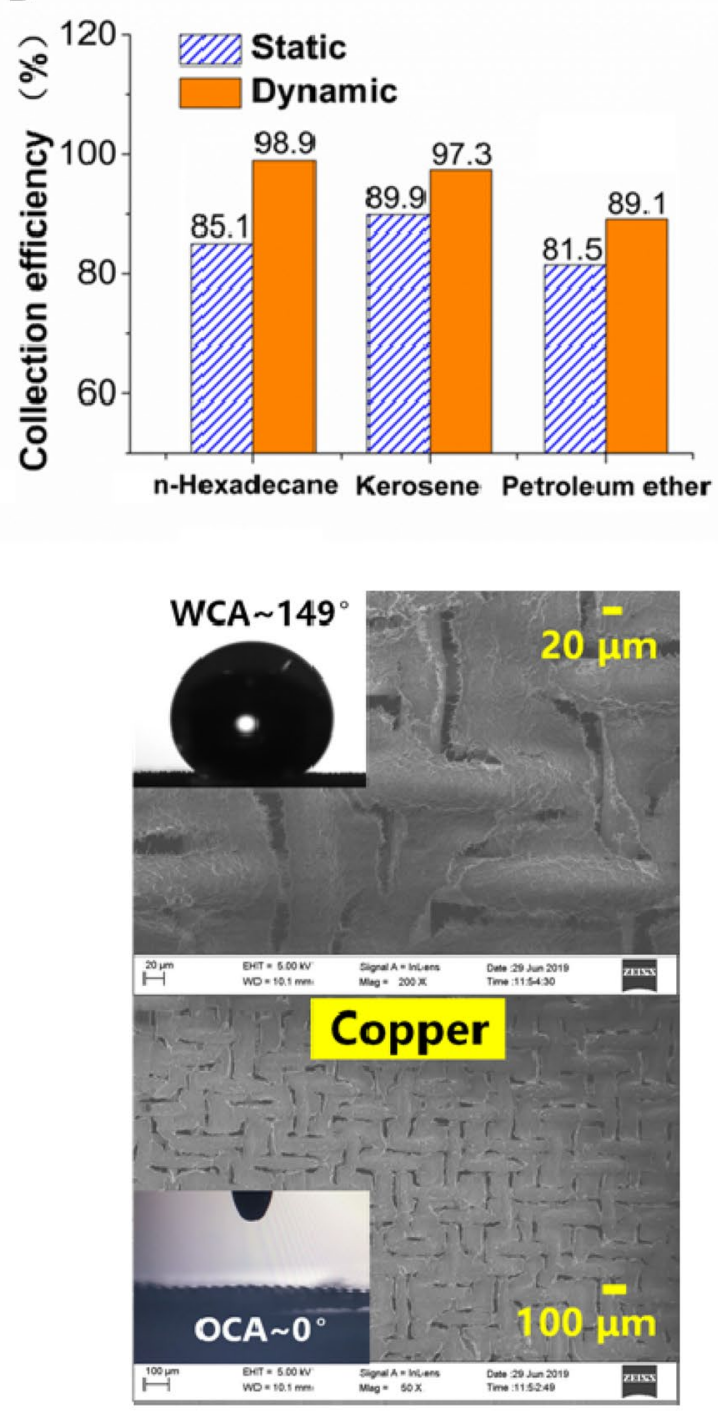

a contact angle of $149^{\circ}$ and OCA remained $0^{\circ}$. Figure $6 c$ demonstrates the collection efficiency $(\eta)$ and thickness of oil slick versus mass of added oil $(65,105,145$ and $185 \mathrm{~g})$. The collection efficiency, $\eta$, was calculated as follows: 
4Fig. 6 a Effect of mesh number on the oil collection using a CTCshaped collector (inclination angle of $60^{\circ}$ ) with $\mathrm{PVDF} / \mathrm{SiO}_{2}$-coated meshes. $\mathbf{b}$ Mass of the collected oil versus time using a CTC-shaped collector (inclination angle of $60^{\circ}$ ) with $\mathrm{PVDF} / \mathrm{SiO}_{2}$-coated stainless steel (200 mesh) and coated copper mesh (200 mesh), respectively. c The collection efficiency and thickness of oil slick versus mass of successively added $n$-hexadecane $(65,105,145$ and $185 \mathrm{~g}$, respectively). $\mathbf{d}$ The collection efficiency of $n$-hexadecane, kerosene and petroleum ether (150 g before collection) under "dynamic" and "static" water surfaces. e SEM images of the as-prepared PVDF/ $\mathrm{SiO}_{2}$-coated stainless steel meshes (left) and copper meshes (right). Insets are water contact angle (WCA, top) and oil contact angle (OCA, bottom) of the corresponding meshes. The magnifications are $200 \times$ (upper inset) and 50 x (lower inset) perspectively. Voltage used for every SEM image is equally $5 \mathrm{kV}$

$\eta=\frac{m_{p}}{m_{o}} \times 100 \%$

where $m_{0}$ and $m_{\mathrm{p}}$ are the mass of the oil before and after the collection, respectively $[22,36]$. The average collection efficiency over $90 \%$ is obtained for all levels of the oil slick, and with the increase of the oil amount (or thickness of oil slick) the efficiency goes up but never reaches $100 \%$, which is due to the oil adsorbed and stuck to the coated meshes (as indicated by SEM in Fig. 6e) and the collector, the loss during the oil transportation, and some residuals of oil on the water surface. Figure $6 \mathrm{~d}$ shows the collection efficiency $(\eta)$ of the same amount $(150 \mathrm{~g}$ before collection) of light oils (petroleum ether, kerosene, and $n$-hexadecane) under "dynamic" and "static" water surface. The collection efficiency under "dynamic" condition is normally around $10 \%$ higher than "static" condition regardless of the oil type. It is noted that petroleum ether shows the lowest collection efficiency (less than 90\% even under "dynamic" condition), since it has greater volatility than the other two oils.

Compared with the existing mesh-based oil collecting devices, our proposed device is more promising to get real-life applications, in that not only the CTC-shaped collectors improve the collection efficiency and show higher stability and discharge of the collected oils, but also their frameworks can be precisely produced in batch via commercially available 3D printing technology. By adding lifting tables or buoys, the collector can stably float on surface of oil/water mixture and swiftly move toward/around oil slicks by traction of a machine or labor on a ship, which can realize in situ oil collection without limitation of location. Apart from that, newlydeveloped substrate materials can be easily applied onto our designed devices which can achieve more functions and environmental adaptabilities [37-39]. Hence, so long as high-performance superwetting meshes have been developed, our proposed engineering device is believed to receive great interest for tackling the worldwide problems in oil spills, oily waste water, and water scarcity, etc. [22].

\section{Conclusion}

The importance of the configuration of (super)hydrophobic meshes based oil-collecting device has been demonstrated for the oil collection. We have verified that a proper design of the (super)hydrophobic meshes based oil-collecting device, e.g. CTC-shape collectors with a small angle of $30^{\circ}$ in this work, not only improves the oil collection efficiency, but also facilitates the evacuation of temporarily collected oils in spite of little amount of the oils and enables the device to keep stable in practical oil collection.

Acknowledgements This work was supported by Natural Science Foundation of Fujian Province (No. 2020J01709), Distinguished Young Scholars of Fujian Province under Grant (No.2018J06014), Youth Innovation Fund of Xiamen City (No. 3502Z20206010) and Subsidized Project for Postgraduates' Innovative Fund in Scientific Research of Huaqiao University (No.18014080028).

Data availability All data generated or analysed during this study are included in this published article.

\section{Compliance with ethical standards}

Conflict of interest The authors declare that they have no conflict of interest.

Open Access This article is licensed under a Creative Commons Attribution 4.0 International License, which permits use, sharing, adaptation, distribution and reproduction in any medium or format, as long as you give appropriate credit to the original author(s) and the source, provide a link to the Creative Commons licence, and indicate if changes were made. The images or other third party material in this article are included in the article's Creative Commons licence, unless indicated otherwise in a credit line to the material. If material is not included in the article's Creative Commons licence and your intended use is not permitted by statutory regulation or exceeds the permitted use, you will need to obtain permission directly from the copyright holder. To view a copy of this licence, visit http://creativecommons .org/licenses/by/4.0/.

\section{References}

1. Wang B et al (2015) Biomimetic super-lyophobic and super-lyophilic materials applied for oil/water separation: a new strategy beyond nature. Chem Soc Rev 44(1):336-361

2. Li J et al (2018) Polymeric materials with switchable superwettability for controllable oil/water separation: a comprehensive review. Prog Polym Sci 87:1-33

3. Bullock RJ et al (2019) In-situ burning with chemical herders for Arctic oil spill response: meta-analysis and review. Sci Total Environ 675:705-716 
4. Farooq $U$ et al (2018) Study of the oil interaction towards oil spill recovery skimmer material: effect of the oil weathering and emulsification properties. Mar Pollut Bull 135:119-128

5. Liu C et al (2018) Oil recovery from tank bottom sludge using rhamnolipids. J Pet Sci Eng 170:14-20

6. Talimi $V$ et al (2019) Enhancing harsh environment oil spill recovery using air floatation system. Saf Extrem Environ 1(1):27-37

7. Rosenblum JS et al (2016) Hydraulic fracturing wastewater treatment by coagulation-adsorption for removal of organic compounds and turbidity. J Environ Chem Eng 4(2):1978-1984

8. Ma W et al (2020) Biomimetic durable multifunctional selfcleaning nanofibrous membrane with outstanding oil/water separation, photodegradation of organic contaminants, and antibacterial performances. ACS Appl Mater Interfaces 12(31):34999-35010

9. Khosravi M et al (2019) Efficient oil/water separation by superhydrophobic CuxS coated on copper mesh. Sep Purif Technol 215:573-581

10. Sun $Y$ et al (2020) Surface hydrophilic modification of PVDF membranes based on tannin and zwitterionic substance towards effective oil-in-water emulsion separation. Sep Purif Technol 234:116015

11. Feng $L$ et al (2004) A superhydrophobic and superoleophilic coating mesh film for the separation of oil and water. Angewandte Chemie 116(15):2046-2048

12. Pham VH et al (2014) Superhydrophobic silanized melamine sponges as high efficiency oil absorbent materials. ACS Appl Mater Interfaces 6(16):14181-14188

13. Zhu Q et al (2011) Facile removal and collection of oils from water surfaces through superhydrophobic and superoleophilic sponges. J Phys Chem C 115(35):17464-17470

14. Su C et al (2019) Highly hydrophobic and oleophilic foam for selective absorption. Appl Surf Sci 256(5):1413-1418

15. Eum KY et al (2019) Superhydrophobic and superoleophilic nickel foam for oil/water separation. Korean J Chem Eng 36(8):1313-1320

16. Jiang ZR et al (2016) Coating sponge with a hydrophobic porous coordination polymer containing a low-energy CF 3-decorated surface for continuous pumping recovery of an oil spill from water. NPG Asia Mater 8(3):e253

17. Liu Y et al (2018) On-demand oil/water separation of 3D Fe foam by controllable wettability. Chem Eng J 331:278-289

18. Wang CF et al (2013) Robust superhydrophobic/superoleophilic sponge for effective continuous absorption and expulsion of oil pollutants from water. ACS Appl Mater Interfaces 5(18):8861-8864

19. Xie A et al (2019) Facile and green fabrication of superhydrophobic sponge for continuous oil/water separation from harsh environments. Coll Surf A Physicochem Eng Asp 563:120-129

20. Lu $Y$ et al (2014) Creating superhydrophobic mild steel surfaces for water proofing and oil-water separation. J Mater Chem A 2(30):11628-11634

21. Li J et al (2018) Continuous, high-flux and efficient oil/water separation assisted by an integrated system with opposite wettability. Appl Surf Sci 433:374-380

22. Lei $T$ et al (2020) 2D $\rightarrow 3 \mathrm{D}$ conversion of superwetting mesh: a simple but powerful strategy for effective and efficient oil/water separation. Sep Purif Technol 242:116244
23. Cheng $M$ et al (2011) A functionally integrated device for effective and facile oil spill cleanup. Langmuir 27(12):7371-7375

24. Wang F et al (2014) Superhydrophobic and superoleophilic miniature device for the collection of oils from water surfaces. J Phys Chem C 118(12):6344-6351

25. Deng $D$ et al (2013) Hydrophobic meshes for oil spill recovery devices. ACS Appl Mater Interfaces 5(3):774-781

26. Ju G et al (2016) Magnetically driven functionally integrated device for continuous and efficient collection of oil droplets from water. RSC Adv 6(25):20559-20564

27. Song J et al (2014) Self-driven one-step oil removal from oil spill on water via selective-wettability steel mesh. ACS Appl Mater Interfaces 6(22):19858-19865

28. Yu Z et al (2015) Multilayer three-dimensional structure made of modified stainless steel mesh for in situ continuous separation of spilled oil. Ind Eng Chem Res 54(47):11838-11843

29. Boticas I et al (2019) Superhydrophobic cotton fabrics based on ZnO nanoparticles functionalization. SN Appl Sci 1(11):1376

30. Cai $Y$ et al (2019) Superhydrophobic metal-organic framework membrane with self-repairing for high-efficiency oil/water emulsion separation. ACS Sustain Chem Eng 7(2):2709-2717

31. Mi HY et al (2018) Superhydrophobic graphene/cellulose/silica aerogel with hierarchical structure as superabsorbers for high efficiency selective oil absorption and recovery. Ind Eng Chem Res 57(5):1745-1755

32. Cai $X$ et al (2017) A critical analysis of the $\alpha, \beta$ and $\gamma$ phases in poly (vinylidene fluoride) using FTIR. RSC Adv 7(25):15382-15389

33. Lei T et al (2018) New insight into gap electrospinning: toward meter-long aligned nanofibers. Langmuir 34(45):13788-13793

34. Finnegan $\mathrm{W}$ et al (2013) The wave excitation forces on a truncated vertical cylinder in water of infinite depth. J Fluids Struct 40:201-213

35. Crick CR et al (2013) Superhydrophobic polymer-coated coppermesh; membranes for highly efficient oil-water separation. J Mater Chem A 1(19):5943-5948

36. Jiang $S$ et al (2020) Electrospinning superhydrophobic-superoleophilic PVDF-SiO ${ }_{2}$ nanofibers membrane for oil-water separation. J Appl Polym Sci 137(47):49546

37. Latthe SS et al (2019) Superhydrophobic leaf mesh decorated with $\mathrm{SiO}_{2}$ nanoparticle-polystyrene nanocomposite for oilwater separation. ACS Appl Nano Mater 2(2):799-805

38. Kang $L$ et al (2019) A new architecture of super-hydrophilic $\beta$-SiAION/graphene oxide ceramic membrane for enhanced anti-fouling and separation of water/oil emulsion. Ceram Int 45(13):16717-16721

39. Sun J et al (2020) A low cost paper tissue-based $\mathrm{PDMS} / \mathrm{SiO}_{2}$ composite for both high efficient oil absorption and water-inoil emulsion separation. J Clean Prod 244:118814

Publisher's Note Springer Nature remains neutral with regard to jurisdictional claims in published maps and institutional affiliations. 